\title{
Biological Water Treatment Residuals from Plants Quinoa Processors
}

\section{Tratamiento Biológico de Aguas Residuales Provenientes de Plantas Procesadoras de Quinua}

\author{
Livaneza Concepción Bayas, Mabel Mariela Parada, and Hugo Segundo \\ Calderón
}

Escuela Superior Politécnica de Chimborazo, Facultad de Ciencias, Carrera de Ingeniería

I International Congress of

Biotechnology, Environment, Chemistry and Food

Corresponding Author:

Livaneza Concepción Bayas

livaneza.bayas@espoch.edu.ec

Published: 29 August 2021

Production and Hosting by

Knowledge $E$

(c) Livaneza Concepción Bayas et al. This article is distributed under the terms of the Creative Commons Attribution License, which permits unrestricted use and redistribution provided that the original author and source are credited. 
sedimentador secundario y por último un filtro de antracita y arena; de esta manera se logró obtener un agua tratada que cumple con la legislación actual para la descarga de efluentes a un cuerpo de agua dulce con un porcentaje de remoción de contaminantes del 97\% y dando la pauta para que incluso el agua pueda ser recirculada en el mismo proceso o reutilizada para otro fin pertinente.

Palabras Clave: Ingeniería química, tratamiento biológico, lavado de quinua, índice de biodegradabilidad, parámetros contaminantes

\section{Introducción}

Los gobiernos intentan obligar a quienes contaminan a que arreglen los efectos negativos que provocan sobre el medio ambiente [1]. Tras procesar la quinua para su comercialización se generan aguas residuales que son desechadas al medio ambiente sin tratamiento previo alguno. Dicho procesamiento implica un desaponificado de la quinua el mismo que consiste en aplicar turbulencia en forma circular al grano de quinua para eliminar la saponina de éste [2]. Como consecuencia del desaponificado de la quinua se generan efluentes con altas concentraciones de saponina que se desechan a acequias, manantiales y cursos de agua libremente ignorando su alta carga contaminante, la saponina que se encuentra en solución es muy tóxica para animales de agua fría como peces, moluscos, sapos, ranas y otros debido a que permeabiliza sus membranas respiratorias y causa su muerte [3]. La toxicidad de este tipo de aguas residuales tiene un alto impacto en ecosistemas frágiles como el altiplano donde generalmente se cultiva y procesa la quinua en mayor cantidad [4].

Ecuador, al ser exportador de quinua se ve en la necesidad de tener una excelente calidad en sus productos y una continua innovación en sus procesos. Dicho esto, se ha generado gran preocupación en las plantas procesadoras de quinua ya que no dan ningún tratamiento al agua residual con altas concentraciones de saponina y simplemente la desechan generando inconformidades en auditorías ambientales y ocasionando posibles paras de producción, además del poco aprovechamiento que le estarían dando al agua que se utiliza en gran cantidad para el lavado de quinua y que tranquilamente podría ser recirculada o reutilizada en cualquier fin pertinente previo su tratamiento [5].

Uno de los principales contaminantes en aguas residuales son los tensoactivos conocidos también como agentes químicos de superficie activa que se encuentran en productos industriales como detergentes, pinturas, cosméticos entre otros: Son unos de los principales contaminantes de aguas residuales [6]; pero no todos los tensoactivos son industrializados como los citados anteriormente, también existen surfactantes naturales como las saponinas [7]. Como resultado de los efectos contaminantes de los tensoactivos se tiene aumento de $\mathrm{pH}$, aumento de nutrientes en los cauces de agua que se depositan promoviendo la formación de algas, se pueden disolver en el agua metales pesados que generan mutaciones en especies, cloro y compuestos organoclorados suelen aumentar su concentración. Según estudios previos realizados 
los puntos antes tratados afectan a parámetros como: Tensoactivos, demanda química de oxígeno, demanda bioquímica de oxígeno, grasas y aceites, hidrocarburos totales, compuestos fenólicos y otros, sobrepasando los límites permisibles establecidos en la norma ambiental, superando en algunos de los casos, con concentraciones muy elevadas [8]. Pese a que la saponina tiene múltiples aplicaciones no se conoce de un tratamiento que separe a la misma del efluente. Sin embargo, de manera generalizada se sabe de tratamientos físicos que pueden ser combinados con tratamientos biológicos o químicos dependiendo de las características que tenga el agua residual a tratar [9].

El presente trabajo tiene como objetivo aplicar un tratamiento biológico a las aguas residuales provenientes de plantas procesadoras de quinua para que cumplan con la norma de calidad ambiental vigente para su descarga y si es posible recircular y reutilizar el agua tratada. Los tratamientos biológicos tienen mayores rendimientos con menores costos económicos de explotación y mantenimiento, y combaten casi en su totalidad a los contaminantes, transformándolos en sustancias inocuas como el dióxido de carbono, metano, nitrógeno molecular y el agua; la mineralización de compuestos mediante microorganismos es entonces un proceso destructivo completo [10].

\section{Materiales y Métodos}

La toma y almacenamiento de las muestras de agua residual se hizo bajo la normativa NTE INEN 2176: 2013 [11] y NTE INEN 2169: 2013 [12] en la planta procesadora de quinua COPROBICH, ubicada en el cantón Colta, perteneciente a la provincia de Chimborazo.

La caracterización fisicoquímica y microbiológica se llevó a cabo en el laboratorio de calidad de agua de la ESPOCH con procedimientos establecidos en los métodos normalizados para el análisis de aguas potables y residuales: APHA [13], AWWA [14], WPCF [15], STANDARD METHODS $21^{\circ}$ EDICIÓN [16] y métodos $\mathrm{HACH}$ [17] adaptados del STANDARD METHODS $21^{\circ}$ EDICIÓN.

Los valores obtenidos de la caracterización antes mencionada fueron comparados con los límites de descarga a un cuerpo de agua dulce establecidos en la actualización hasta el año 2019 de la NORMA DE CALIDAD AMBIENTAL Y DESCARGA DE EFLUENTES: RECURSO AGUA [18] para establecer los parámetros que se encuentran fuera de la normativa vigente y así trabajar en base a éstos (Tabla 1).

Los procesos que se emplearon para dar tratamiento al agua residual se obtuvieron de la experimentación a nivel de laboratorio, siendo éstos detallados a continuación:

\subsection{Sedimentación primaria}

Debido al alto contenido de sólidos sedimentables en el agua residual, el primer proceso al que fue sometida dicha agua fue una sedimentación primaria durante $2 \mathrm{hr}$ en la cual se separaron los sólidos de mayor tamaño. Los tanques de sedimentación primaria bien dimensionados eliminan 50 a 70 por 100 de los sólidos finamente divididos, y entre 25 a 40 por 100 de $\mathrm{DBO}_{5}[20]$. 


\section{Table 1}

Análisis comparativo de los valores obtenidos de la caracterización fisicoquímica y microbiológica del agua residual con la normativa vigente.

\begin{tabular}{|c|c|c|c|}
\hline Parámetros & Unidad & Muestra agua residual & Límite max. \\
\hline Aceites y grasas & $\mathrm{mg} / \mathrm{L}$ & 9 & 0,3 \\
\hline Color real & Unds de Pt/Co & 3520 & $\begin{array}{l}\text { Inapreciable en dilución: } \\
1 / 20\end{array}$ \\
\hline Turbiedad & NTU & 931 & $\ldots \ldots \ldots \ldots .$. \\
\hline Conductividad & uS & 1308 & $\ldots \ldots \ldots \ldots$ \\
\hline $\mathrm{pH}$ & & 8,3 & $5-9$ \\
\hline Color aparente & Unds de Pt/Co & 3520 & $\ldots \ldots \ldots \ldots$. \\
\hline $\begin{array}{l}\text { Sólidos suspendidos } \\
\text { totales }\end{array}$ & $\mathrm{mg} / \mathrm{L}$ & 1195 & 100 \\
\hline Temperatura & ${ }^{\circ} \mathrm{C}$ & 19 & $<35$ \\
\hline $\begin{array}{l}\text { Sólidos } \\
\text { sedimentables }\end{array}$ & $\mathrm{mg} / \mathrm{L}$ & 13500 & 1 \\
\hline Sólidos totales & $\mathrm{mg} / \mathrm{L}$ & 4644 & 1600 \\
\hline Nitrógeno total & $\mathrm{mg} / \mathrm{L}$ & 250 & 15 \\
\hline DQO & $\mathrm{mg} / \mathrm{L}$ & 5000 & 250 \\
\hline DBO5 & $\mathrm{mg} / \mathrm{L}$ & 2990 & 100 \\
\hline Coliformes fecales & UFC/100 ml & 240 & Remoción > al 99,9% \\
\hline Tensoactivos & $\mathrm{mg} / \mathrm{L}$ & 17,6 & 0,5 \\
\hline
\end{tabular}

pH: Potencial de hidrógeno; DQO: Demanda química de oxígeno; DBO5: Demanda bioquímica de oxígeno.

Table 2

Índice de biodegradabilidad.

DBO5/DQO
$>0,5$
$0,2-0,5$
$<0,2$

Biodegradabilidad
Muy biodegradable
Biodegradable
Poco biodegradable

Tipo de tratamiento
Tratamiento biológico
Tratamiento biológico o fisicoquímico
Tratamiento fisicoquímico

DQO: Demanda química de oxígeno; DBO5: Demanda bioquímica de oxígeno.

\subsection{Aireación (reactor biológico)}

Este proceso es de vital importancia, ya que con éste se pretende eliminar la mayor cantidad de carga orgánica del agua residual y bajar los parámetros contaminantes en una gran proporción.

Para llevar a cabo la aireación se utilizó la muestra previamente sedimentada a la que se inyectó aire para mantener el agua en turbulencia con ayuda de una bomba y difusores, de esta manera se favoreció al crecimiento microbiano. Dichos organismos son los encargados de asimilar la materia orgánica formando flóculos [21]. La aireación se la realizó durante tres días ( $72 \mathrm{hr}$ ) ya que a partir de los tres días es que se consiguió buenos resultados. 
Cabe destacar que la aireación fue muy lenta y utilizando unos difusores muy pequeños debido a que se genera una gran cantidad de espuma por la presencia de tensoactivos naturales en la muestra de agua residual [22]

\subsection{Sedimentación secundaria}

Una vez culminada la aireación de la muestra de agua residual se procedió a realizar una nueva sedimentación por gravedad durante $5 \mathrm{hr}$ para que la gran cantidad de materia orgánica formada en la aireación pueda precipitar por completo. las partículas aglomerables (materia orgánica que logra flocularse o hacerse grumos), se sedimenta cuando ganan el peso y se precipitan a la velocidad creciente en el tiempo [23].

\subsection{Filtración}

Por último, para alcanzar los límites establecidos en la normativa vigente se llevó a cabo una filtración para bajar en una mayor proporción la carga orgánica aún presente en la muestra de agua aireada.

El filtro que se utilizó para la última etapa de tratamiento estaba compuesto por arena y antracita y la filtración se realizó con bajos volúmenes de agua, muy lenta y controlada.

\section{Resultados}

Se detallan los resultados obtenidos de la caracterización fisicoquímica y microbiológica del agua tratada a nivel de laboratorio los cuales también fueron comparados con los límites de descarga a un cuerpo de agua dulce establecidos en la normativa vigente con la finalidad corroborar la disminución en los valores obtenidos en un inicio y el porcentaje de remoción de los parámetros que en un inicio se encontraban fuera de norma y por tanto eran considerados como contaminantes (Tablas 3 y 4).

\section{Discusión}

En los valores obtenidos de la primera caracterización (Tabla 1) se encontró una demanda bioquímica y química de oxígeno bastante elevada puesto que se trata del lavado de quinua la cual contiene una gran cantidad de saponinas mismas que se comportan como surfactantes naturales y alteran parámetros como: tensoactivos, demanda química de oxígeno, demanda bioquímica de oxígeno, grasas y aceites y otros [7], se encontró además una considerable presencia de nitrógeno debido al valor nutricional de la quinua; también no es raro que se hayan encontrado una gran cantidad de sólidos suspendidos, sedimentables y totales por el escarificado previo que sufre la quinua; otros parámetros que se encontraron fuera del límite permisible eran las grasas y los tensoactivos que también se justifica por lo mencionado antes acerca del comportamiento de la saponina como un tensoactivo natural. 
Table 3

Comparación de resultados obtenidos la caracterización fisicoquímica y microbiológica del agua tratada con la normativa vigente.

\begin{tabular}{|c|c|c|c|}
\hline Parametros & Unidad & Muestra tratada & Límite máx. \\
\hline Aceites y grasas & $\mathrm{mg} / \mathrm{l}$ & 0,1 & 0,3 \\
\hline Color real & Unds de Pt//Co & 30 & Inapreciable en dilución: 1/20 \\
\hline Turbiedad & NTU & 13,77 & .............. \\
\hline Conductividad & uS & 580 & .............. \\
\hline $\mathrm{pH}$ & & 7,71 & $6-9$ \\
\hline Sólidos suspendidos totales & $\mathrm{mg} / \mathrm{l}$ & 7 & 100 \\
\hline Temperatura & ${ }^{\circ} \mathrm{C}$ & 21,8 & $<35$ \\
\hline Sólidos sedimentables & $\mathrm{mg} / \mathrm{l}$ & Ausencia & 1 \\
\hline Sólidos totales & $\mathrm{mg} / \mathrm{l}$ & 392 & 1600 \\
\hline N Total & $\mathrm{mg} / \mathrm{l}$ & 5 & 15 \\
\hline DQO & $\mathrm{mg} / \mathrm{l}$ & 224 & 250 \\
\hline DBO5 & $\mathrm{mg} / \mathrm{l}$ & 94 & 100 \\
\hline Coliformes fecales & UFC/100ml & Ausencia & Remoción > al 99,9\% \\
\hline Tensoactivos & $\mathrm{mg} / \mathrm{l}$ & 0,16 & 0,5 \\
\hline
\end{tabular}

pH: Potencial de hidrógeno; DQO: Demanda química de oxígeno; DBO5: Demanda bioquímica de oxígeno.

Table 4

Porcentaje de remoción de parámetros contaminantes.

\begin{tabular}{l|l|l|l|l} 
Parámetros & Unidad & Muestra agua residual & Muestra tratada & \% Remoción \\
\hline $\begin{array}{l}\text { Aceites y grasas } \\
\text { Sólidos suspendidos }\end{array}$ & $\mathrm{mg} / \mathrm{l}$ & 9 & 0,1 & 98,9 \\
\hline $\begin{array}{l}\mathrm{mg} / \mathrm{l} \\
\text { Sotales }\end{array}$ & 1195 & 7 & 99,4 \\
\hline Sólidos sedimentables & $\mathrm{mg} / \mathrm{l}$ & 13500 & 0 & 100,0 \\
\hline Sólidos totales & $\mathrm{mg} / \mathrm{l}$ & 4644 & 392 & 91,6 \\
\hline Nitrógeno total & $\mathrm{mg} / \mathrm{l}$ & 250 & 5 & 98,0 \\
\hline DQO & $\mathrm{mg} / \mathrm{l}$ & 5000 & 224 & 95,5 \\
\hline DBO5 & $\mathrm{mg} / \mathrm{l}$ & 2990 & 94 & 96,9 \\
\hline Tensoactivos & $\mathrm{mg} / \mathrm{l}$ & 17,6 & 0,16 & 99,1 \\
\hline
\end{tabular}

DQO: Demanda química de oxígeno; DBO5: Demanda bioquímica de oxígeno.

El índice de biodegradabilidad de la muestra de agua residual tuvo un valor igual a 0,6 lo que quiere decir que es muy biodegradable (Tabla 2) y por ende se pudo realizar un tratamiento biológico para la depuración de su carga contaminante; dicho valor fue muy conveniente ya que uno de los planteamientos iniciales a más de cumplir con la normativa vigente fue el recircular el agua para el mismo proceso; para ello el agua no podía ser tratada con ningún químico puesto que estas empresas manejan normativas de exportación para las cuales deben cumplir con una certificación orgánica. 
Se destaca que los procesos de aireación y filtrado fueron los principales responsables de la remoción de parámetros contaminantes ya que se evidenció una gran reducción en los mismos durante estos dos procesos; además existen algunas pruebas para identificar cualitativamente las saponinas, una de ellas se la conoce como prueba de espuma según Flores, Huamán y Tomás en su artículo publicado en la Revista Peruana de Química e Ingeniería Química 'ESTUDIO COMPARATIVO DE TRES METODOLOGÍAS CUANTITATIVAS DE EXTRACCIÓN DE SAPONINAS DE LA MELISA OFFICINALIS "TORONJIL"' [23], dichas pruebas se realizaron antes y después de los procesos de aireación y filtrado para corroborar la efectiva disminución de la carga orgánica. Es importante mencionar que la filtración tuvo un gran papel ya que no se realizó una aireación prolongada por dos razones, la primera porque al ser un agua con tensoactivos naturales se generaba demasiada espuma y la segunda porque la muestra de agua a los tres días de aireación empezó a dar muy buenos resultados esto seguramente se debe a que el proceso en donde se tomó la muestra de agua utilizaba agua de pozo y no potable, puesto que esta última generalmente no tiene ningún tipo de microorganismos además de la presencia de nitrógeno (Tabla 1), principal nutriente de microorganismos en dicha muestra de agua por lo que se justificaría la eficaz aparición de microorganismos.

Según Julián Quiroga y Fabio López en su artículo publicado en la Revista de Tecnología 'Tratamiento de aguas residuales mediante lodos activados a escala laboratorio', menciona que: El oxígeno cumple un importante papel en el tratamiento biológico de aguas residuales, esto debido a que tanto su ausencia como presencia condiciona el tipo de organismos que se encargarán de eliminar o degradar la materia orgánica presente en el agua residual [24]. Dicho esto se puede resaltar que la aireación realizada al agua residual tuvo una considerable influencia en el tratamiento biológico realizado ya que se obtuvo un porcentaje de remoción de contaminantes de 97,4\% (Tabla 4). Cabe destacar que los procesos empleados para el tratamiento biológico fueron validados exclusivamente por el cumplimiento de los parámetros con la normativa vigente (Tabla 3) y por la disminución de los parámetros contaminantes en cada uno de los mismos y principalmente con la comparación de la caracterización inicial y final de la muestra de agua tomada para realizarse dicho tratamiento (Tabla 4), asegurando de esta manera su gran eficiencia.

\section{Conclusiones}

Este estudio es de suma importancia ya que constituye uno de los primeros en el que se logra realizar un tratamiento biológico de aguas residuales provenientes del lavado de quinua en plantas procesadoras de la misma, cumpliendo de esta manera con la normativa vigente para descarga de efluentes en un cuerpo de agua dulce establecidos en su actualización hasta el año 2019 y obteniendo un porcentaje de remoción de carga contaminante igual al 97,4\% un valor bastante bueno y aceptable. Además, se aprovecha al máximo el recurso agua ya que se recomienda la recirculación del agua tratada o su reutilización para fines pertinentes. 
Cabe destacar que en estudios posteriores es necesario analizar los lodos generados como subproducto a lo largo del tratamiento ya que tienen una alta concentración de saponinas, mismas que son muy apetecidas comercialmente para la elaboración de otros productos como shampoo, jabón, pasta dental, insecticidas, entre otros.

\section{Agradecimientos}

Se destaca el aporte tan indispensable y necesario por parte de la Corporación de Productores y Comercializadores Orgánicos Bio Taita Chimborazo (COPROBICH) y se extiende de manera muy especial un sincero agradecimiento por su buena acogida y su presta colaboración.

\section{References}

[1] Moreno E. La experiencia de Holanda y Alemania en el uso de cargos por vertimientos de aguas residuales como instrumento para el control de la contaminación hídrica Elementos para decidir los parámetros a ser. Economía y Desarrollo 2005;4(1):57-83.

[2] Nieto C, Vimos C, Monteros Caicedo C, Rivera M. NIAP-Ingapirca e INIAP-Tunkahuan: Dos variedades de quinua de bajo contenido de saponina. INIAP, Estación Experimental Santa Catalina, Programa de Cultivos Andinos 1992;288:20.

[3] Guamarra M, Aguilar I. La adopción de tecnología como una forma de internalizar las externalidades ambientales del beneficiado de quinua en Oruro Bolivia [Internet]. Monterrey: CONACYT; 2016. Disponible de: https://www.colef.mx/posgrado/wp-content/uploads/2016/12/ TESIS-Del-Barco-Gamarra-Mar\%C3\%ADa-Teresa.pdf

[4] Echalar A, Torrico J. Consecuencias del incremento de la producción de quinua (Chenopodium quinoa Willd.) en el Altiplano Sur de Bolivia. Ciencia Agronómica 2015;1:29-34.

[5] Villaroel J, García E. Optimización del proceso de desaponificado de la quinua por el método de lavado, mediante la implementación de una lavadora industrial en la empresa Asoalienu [Tesis]. Riobamba: Escuela Ssuperior Politécnica de Chimborazo; 2019.

[6] Usiña K, Borja P. Análisis de las propiedades surfactantes de saponinas obtenidas de los frutos de Sapindus Saponaria L[Tesis]. Quito: Universidad Central del Ecuador; 2017.

[7] Rodriguez J. Monar G. Determinación y cuantificación de saponinas en las hojas de la cabuya (furcraea andina) para su posible uso como tensoactivo en detergentes biodegradables [Tesis]. Guayaquil: Universidad de Guayaquil; 2017.

[8] Brand D. Efectos de los tensoactivos en el medio ambiente [Internet]. California: Universidad de Santiago de Cali; 2019. Disponible de: https://repository.usc.edu.co/bitstream/20.500.12421/623/1/ EFECTOS\%20DE\%2OLOS\%20TENSOACTIVOS.pdf

[9] Romero J. Tratamiento de aguas residuales, teoría y principios de diseño [Internet]. Bogotá: Escuela Colombiana de Ingenieros; 2004. Disponible de: http://dspace.ucbscz.edu.bo/dspace/bitstream/ 123456789/22876/1/11991.pdf

[10] Anáiz C. Tratamiento biológico de aguas residuales. Revista Tecnológica del Agua [Internet]. 2000. Disponible de: https://cidta.usal.es/cursos/etap/modulos/libros/edar.pdf

[11] INEN. Norma Técnica Ecuatoriana NTE INEN 2 176; 2013.

[12] INEN. Norma Técnica Ecuatoriana NTE INEN 2 169.1998. Agua. Calidad del agua. Muestreo. Manejo y conservación de muestras. Quito-Ecuador; 2013.

[13] Torres S, de Navia S. Calidad fisicoquímica y microbiológica del agua del municipio de Bojacá, Cundinamarca [Internet]. 2010 [Consultado 2019 Nov 24]. Disponible de: https://cidta.usal.es/cursos/ etap/modulos/libros/edar.pdf

[14] AWWA. Norma ANSI. AWWA C654-87. Desinfección de pozos; 1987.

[15] SANEAMENTO AMBIENTAL. Companhia de Tecnología [Internet]. 2016 [Consultado 2019 Dic 12] Disponible de: https://www.cetesb.sp.gov.br

[16] Van H. Current situation on regulations for mycotoxins. Overview of tolerances and status of standard methods of sampling and analysis. Food Additives \& Contaminants 1989;6(2):139-188.

[17] Sosa de Martínez M, Pablos H, Santos A. Guía para elaborar el protocolo de investigación: IV. 1995;21. 
[18] Presidencia de la Republica. Norma de calidad ambiental y de descarga de efluentes: Recurso agua [Internet]. [Consultado 2019 Dic 12]. Disponible de: http://extwprlegs1.fao.org/docs/pdf/ecu112180.pdf

[19] Metcalf L, Eddy H. Ingeniería de aguas residuales. Tratamiento, vertido y reutilización. 3rd ed. MadridEspaña: McGraw-Hill; 1995.

[20] Montiel H. Desarrollo de un software para la simulación de sistemas de tratamiento por lodos activados a partir del modelo ASM1 [Internet]. [Consultado 2019 Dic 20]. Disponible de: http://universidadpersonal. net/doc-elec/112817.pdf

[21] Vargas O. Comportamiento de la eficiencia de remoción de materia orgánica en un biorreactor de lecho móvil alimentado con agua residual doméstica, con la variación del volumen del lecho [Internet]. Bogotá, Colombia: Universidad de La Salle; 2016 [Consultado 2019 Dic 20]. Disponible de: https: //ciencia.lasalle.edu.co/cgi/viewcontent.cgi?article=1349\&context=ing_ambiental_sanitaria

[22] Muñoz A. Caracterización y tratamiento de aguas residuales [Internet]. Estado de Hidalgo: Universidad Autónoma del Estado de Hidalgo; 2008. Disponible de: http://universidadpersonal.net/doc-elec/112817. pdf

[23] Uvidia J, Vvillacrés M. Optimización de la planta de tratamiento de agua potable de ciudad de Chambo [Tesis]. Riobamba: Escuela Ssuperior Politécnica de Chimborazo; 2014.

[24] Flores T, Huamán J, Tomás G. Estudio comparativo de tres metodologías cuantitativas de extracción de saponinas de la melisa officinalis "toronjil". Revista Peruana de Química e Ingeniería Química 2013;16(2):47-51.

[25] Quiroga J, López F. Tratamiento de aguas residuales mediante lodos activados a escala laboratorio. Revista de Tecnología 2008;7(2):21-28. 